\title{
Pulmonary hypertension in idiopathic pulmonary fibrosis with mild-to-moderate restriction
}

\author{
Ganesh Raghu' ${ }^{1}$, Steven D. Nathan², Juergen Behr ${ }^{3}$, Kevin K. Brown ${ }^{4}$, \\ James J. Egan ${ }^{5}$, Steven M. Kawut ${ }^{6}$, Kevin R. Flaherty ${ }^{7}$, Fernando J. Martinez ${ }^{7}$, \\ Athol U. Wells ${ }^{8}$, Lixin Shao', Huafeng Zhou' ${ }^{9}$, Noreen Henig ${ }^{10}$, \\ Javier Szwarcberg ${ }^{11}$, Hunter Gillies ${ }^{11}$, Alan B. Montgomery ${ }^{12}$ and \\ Thomas G. O'Riordan ${ }^{13}$
}

\begin{abstract}
Affiliations: 'Dept of Medicine, Division of Pulmonary and Critical Care Medicine, University of Washington, Seattle, WA, USA. ${ }^{2}$ Lung Transplant Program, Inova Fairfax Hospital, Falls Church, VA, USA. ${ }^{3}$ Dept of Internal Medicine V, Comprehensive Pneumology Center, and Asklepios-Clinics-Gauting, Member of the German Center for Lung Research, Germany. ${ }^{4}$ Dept of Medicine, National Jewish Health, Denver, CO, USA. ${ }^{5}$ Dept of Pulmonary Medicine, Mater Misericordiae Hospital, Dublin, Ireland. ${ }^{6}$ Pulmonary Vascular Disease Program, Perelman School of Medicine at the University of Pennsylvania, Philadelphia, PA, USA. ${ }^{7}$ Dept of Internal Medicine, University of Michigan, Ann Arbor, MI, USA. ${ }^{8}$ Interstitial Lung Disease, Royal Brompton Hospital, London, UK. ${ }^{9}$ Dept of Biostatistics, Gilead Sciences Inc., Seattle, WA, USA. ${ }^{10}$ Dept of Medical Affairs, Gilead Sciences Inc., Foster City, CA, USA. ${ }^{11}$ Dept of Clinical Research, Gilead Sciences Inc., Foster City, CA, USA. ${ }^{12}$ Dept of Clinical Research, Cardeas Pharma, Seattle, WA, USA. ${ }^{13}$ Dept of Clinical Research, Gilead Sciences Inc., Seattle, WA, USA.
\end{abstract}

Correspondence: Ganesh Raghu, Center for Interstitial Lung Diseases, UW Medicine, University of Washington Medical Center 1959 N.E. Pacific, Campus Box 356175 Seattle, WA 98195, USA.

E-mail: graghuduw.edu

ABSTRACT The clinical course of pulmonary hypertension (PH) in idiopathic pulmonary fibrosis (IPF) is not known except in advanced disease.

488 subjects in a placebo-controlled study of ambrisentan in IPF with mild-moderate restriction in lung volume, underwent right heart catheterisation (RHC) at baseline and 117 subjects (24\%) had repeated haemodynamic measurements at 48 weeks.

The subjects were categorised into a) World Health Organization (WHO) Group $3 \mathrm{PH}$ (PH associated with pulmonary disease), $\mathrm{n}=68$ (14\%); b) WHO Group $2 \mathrm{PH}$ (PH associated with left-sided cardiac disease), $n=25$ (5\%); c) no PH but elevated pulmonary artery wedge pressure (PAWP), $n=21$ (4\%); and d) no PH but without elevation of PAWP, $\mathrm{n}=374(77 \%)$. The WHO Group $3 \mathrm{PH}$ subjects had a lower diffusion capacity, 6MWD and oxygen saturation compared to the subjects with no $\mathrm{PH}$. There was no significant change in mean pulmonary arterial pressure with ambrisenten or placebo after 12 months.

Subjects with IPF associated with WHO Group $3 \mathrm{PH}$ had impaired gas exchange and exercise capacity compared to patients without $\mathrm{PH}$. An additional $9 \%$ of the subjects had haemodynamic evidence of subclinical left-ventricular dysfunction. Pulmonary artery pressures remained stable over 1 year in the majority of the cohort.

@ERSpublications

In IPF associated with pulmonary hypertension (PH), gas exchange is severely impaired; the $\mathrm{PH}$ was stable over 1 year http://ow.ly/OnvHl

Editorial comment in: Eur Respir J 2015; 46: 1247-1250 [DOI: 10.1183/13993003.01288-2015]

Received: Aug 212014 | Accepted after revision: May 272015 | First published online: Aug 062015

Conflict of interest: Disclosures can be found alongside the online version of this article at erj.ersjournals.com

Copyright OERS 2015 


\section{Introduction}

The clinical course of idiopathic pulmonary fibrosis (IPF) is quite variable [1]. Some patients demonstrate a gradual decline in lung function over several years while, in others, the rate of decline accelerates within months of diagnosis. There may also be periods of indolent decline interrupted by precipitous declines in a "stair-step" pattern of deterioration. Publication of data from prospective clinical trials and retrospective databases have provided insights into the prognostic significance of baseline lung function as well as associated comorbidities, facilitating the phenotyping of patients for future clinical trials of novel therapies. For example, it has been recognised that, if the course of IPF is complicated by pulmonary hypertension $(\mathrm{PH})$, these patients have a worse prognosis than those without $\mathrm{PH}$ [2-6]. $\mathrm{PH}$ has been described in $20-$ $46 \%$ of patients with IPF who undergo right heart catheterisation (RHC) as part of a lung transplant evaluation $[4,5,7]$. In these cases, a diagnosis of $\mathrm{PH}$ was associated with more severe disease as measured by forced vital capacity (FVC), diffusion capacity for carbon monoxide (DLCO) and resting oxygen saturation. Furthermore, in transplant candidates in whom a repeat RHC takes place, the rate of progression of $\mathrm{PH}$ may be rapid [8]. However, patients undergoing lung transplant evaluation may not be representative of the general population with IPF, and routine RHC is not part of the standard care of these patients. Further, interpretation of RHC performed patients about to undergo a transplant surgery is potentially complicated by the effects of positive pressure mechanical ventilation. In a recent IPF multicentre trial (ARTEMIS-IPF) of ambrisentan [9], an endothelin receptor antagonist, in which a RHC was used to stratify subjects on the basis of $\mathrm{PH}$ (mean pulmonary arterial pressure (PAP) $>25 \mathrm{mmHg}$ and pulmonary artery wedge pressure (PAWP) $\leqslant 15 \mathrm{mmHg}$ ), it was demonstrated that $10 \%$ of subjects had $\mathrm{PH}$. Key exclusion criteria for that study included a FVC $<50 \%$ predicted at baseline and extensive honeycombing on high-resolution computerised tomography imaging (HRCT), as well as subjects with New York Heart Association (NYHA) Grade III or IV functional symptoms. In the present analysis, the criteria for diagnosing $\mathrm{PH}$ was changed in accordance with the most recent guidelines of mean PAP $\geqslant 25 \mathrm{mmHg}$ and PAWP $\leqslant 15 \mathrm{mmHg}$ [10]. In addition, we present data on subjects with PAWP $>15 \mathrm{mmHg}$, as an index of subclinical left-ventricular dysfunction in subjects with well-characterised IPF.

The goal of the current paper is to provide further insight into $\mathrm{PH}$ in patients with physiologically and radiographically mild-to-moderate IPF. A unique aspect of the ARTEMIS-IPF study was a protocol-mandated follow-up RHC at 48 weeks which allows the opportunity to evaluate both the incidence and progression of $\mathrm{PH}$ in a group of IPF patients with mild-to-moderate restrictive physiology.

\section{Methods}

Design overview

ARTEMIS-IPF was a randomised, double-blind, placebo-controlled, multi-centred, event-driven phase 3 trial to evaluate whether a selective endothelin-receptor antagonist ambrisentan was effective in improving the progression-free survival (PFS) of patients with mild-to-moderate IPF [9]. PFS was a composite primary endpoint defined as time from randomisation to any one of three protocol defined events: 1) a prespecified decline in FVC and DLCO; 2) an adjudicated respiratory hospitalisation event; or 3) death from any cause. The study was stratified by the presence of $\mathrm{PH}$ at baseline which was defined as a mean PAP $>25 \mathrm{mmHg}$ and a PAWP of $\leqslant 15 \mathrm{mmHg}$. In the present reanalysis, the threshold mean PAP for diagnosis of PH was changed to $\geqslant 25 \mathrm{mmHg}$ to be consistent with current guidelines [10]. Subjects were randomised to placebo or ambrisentan in a 1:2 ratio. The initial dose of ambrisentan was $5 \mathrm{mg}$ once daily for 2 weeks and if tolerated, the dose was increased to $10 \mathrm{mg}$ daily. The protocol specified that all subjects be scheduled to undergo a repeat RHC after 48 weeks therapy or at an early termination visit. This study was approved by local ethics committees and institutional review boards. All subjects provided written, informed consent. After 1087 subjects had been screened (and 494 randomised to study drug), the sponsor terminated the study prematurely due to lack of efficacy and thus by implication, an adverse risk benefit ratio. Once the decision was made to terminate the study, no RHCs were undertaken after that date.

\section{Study participants}

Eligible subjects included both men and women aged 40-80 years who had IPF for 3 months or longer according to the American Thoracic Society/European Respiratory Society guidelines [11]. The diagnosis of IPF had to be supported either by a surgical lung biopsy showing usual interstitial pneumonia or an HRCT classified as "definite usual interstitial pneumonia". All of the HRCT images were reviewed by a member of a centralised panel of radiologists. Similarly surgical lung biopsy slides, when obtained, were reviewed by a member of a central panel of pathologists.

Patients with $\leqslant 5 \%$ honeycombing on HRCT images of the lung were hypothesised to respond to endothelin receptor blockade and thus patients with $>5 \%$ honeycombing on the HRCT were excluded. Other key exclusion criteria included NYHA functional class Grade III or IV symptoms; left-ventricular ejection fraction $<40 \%$; collagen vascular disease; evidence of occupational lung disease, evidence of 
coexisting obstructive airflow defect or prominent emphysema on HRCT; recent hospitalisation or respiratory infection within 60 days; chronic treatment for $\mathrm{PH}$; and chronic treatment with immunosuppressive therapy.

While subjects were allowed to use supplemental oxygen as part of standard care, the use of supplemental oxygen was not permitted during performance of the 6-min walk test (6MWT) and the ability to perform a $6 \mathrm{MWT}$ was an inclusion criterion. Subjects who had an oxygen saturation of $<80 \%$ at rest for 6 consecutive seconds while breathing room air were not permitted to perform the 6MWT and the test was terminated if a subjects demonstrated an oxygen saturation of $<80 \%$ for 6 consecutive seconds during ambulation.

\section{Statistics}

RHC data from subjects were used to classify subjects as per standard diagnostic criteria for PH $[10,12]$ and the groups were compared using Fisher's exact test and Wilcoxon rank sum test for categorical and continuous variables, respectively. Subset analyses were undertaken on subjects with severe $\mathrm{PH}$, using an mean PAP value of $\geqslant 35 \mathrm{mmHg}$ [13]. Comparisons of continuous variables in subjects who underwent a second RHC was undertaken with Spearman's correlation coefficient. Fisher's exact test was used for categorical comparison of the numbers of subjects who developed PH during treatment. Pearson's correlation was used to assess the relationship between mean PAP, FVC, DLCO and 6MWT distance, and Cohen's criteria were used to classify the strength of the relationship [14].

\section{Results}

Subject disposition is shown in a flow chart (figure 1). There were 488 patients randomised who had evaluable RHC data. The demographics and lung function of these subjects with IPF classified into four sub groups based on their haemodynamic profiles are shown in table 1. The four groups were as follows: 1) 68 (14\%) subjects had World Health Organization (WHO) group $3 \mathrm{PH}$ with an elevated mean PAP but a normal PAWP; 2) 25 (5\%) subjects had WHO group 2 PH with both an elevated mean PAP and PAWP; 3 ) a further 21 (4\%) subjects had no PH but with had elevated PAWP; and 4) 374 (77\%) subjects had no PH and without elevation of PAWP. Thus a total of 93 (19\%) of 488 randomised subjects with RHC data had PH at baseline.

Baseline disease characteristics of the four subgroups are shown in table 1 . The p-values shown refer to comparisons of WHO Group 3 subjects to the other three subgroups. For continuous variables, p-values are based on Wilcoxon rank sum test and for categorical variables, p-values are based on Fisher's exact test. The FVC \% predicted at baseline was similar between the four subgroups of subjects. The WHO Group $3 \mathrm{PH}$ subjects had a lower \% predicted DLCO and 6MWD compared with the subjects with no $\mathrm{PH}$

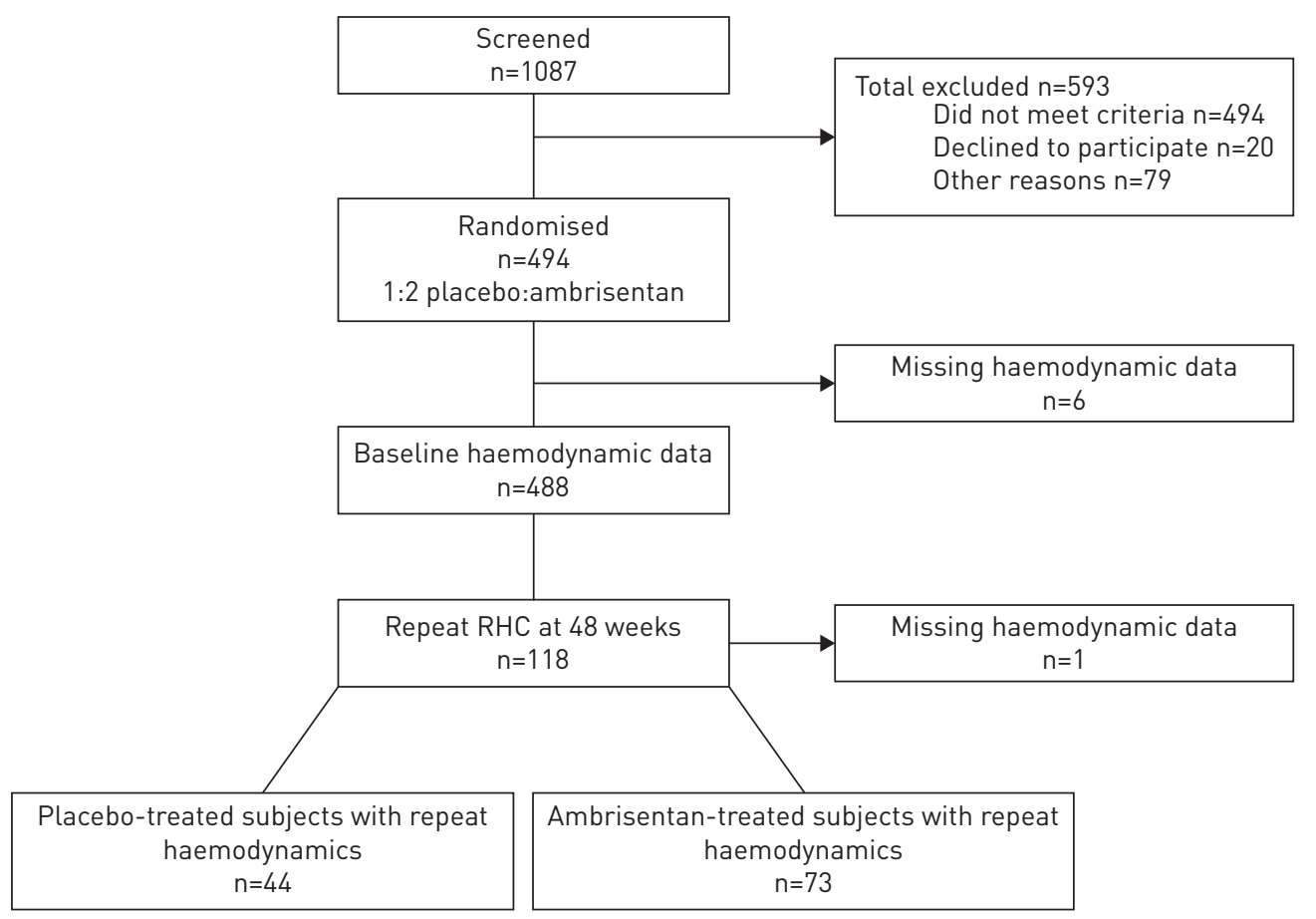

FIGURE 1 Subject disposition. RHC: right-heart catheterisation. 
TABLE 1 Baseline demographic and disease characteristics of subjects with and without pulmonary hypertension (PH) at baseline

Group $3^{\#} \mathrm{PH}$

Group $2^{\#} \mathrm{PH}$

No PH, elevated PAWP

Demographics p-value versus

Demographics

p-value versus

No PH, PAWP not elevated

Group 3 PH

Group $3 \mathrm{PH}$

Demographics p-value versus

Group 3 PH

\begin{tabular}{|c|c|c|c|c|c|c|c|}
\hline Subjects $n$ & 68 & 25 & & 21 & & 374 & \\
\hline Age years & $68.0 \pm 6.14$ & $67.6 \pm 6.28$ & 0.62 & $66.1 \pm 7.65$ & 0.30 & $65.5 \pm 7.44$ & 0.016 \\
\hline Sex male/female & $\begin{array}{l}47(69.1) / \\
21(30.9)\end{array}$ & $\begin{array}{c}20(80.0) / \\
5(20.0)\end{array}$ & 0.44 & $\begin{array}{l}20(81.0) / \\
4(19.0)\end{array}$ & 0.41 & $\begin{array}{l}267(71.4) / \\
107(28.6)\end{array}$ & 0.77 \\
\hline BMI $\mathrm{kg} \cdot \mathrm{m}^{-2}$ & $31.4 \pm 5.12$ & $32.8 \pm 2.87$ & 0.051 & $30.4 \pm 4.81$ & 0.54 & $29.2 \pm 4.38$ & $<0.001$ \\
\hline Smoking status & & & 0.10 & & 0.76 & & 0.78 \\
\hline Never & $22(32.4)$ & $4(16.0)$ & & $5(23.8)$ & & 124 (33.2) & \\
\hline Current & $2(2.9)$ & $3(12.0)$ & & 0 & & $7(1.9)$ & \\
\hline Former & $44(64.7)$ & 18 (72.0) & & $16(76.2)$ & & $243(65.0)$ & \\
\hline Ethnicity & & & 0.32 & & 0.46 & & 0.20 \\
\hline American Indian & $1(1.5)$ & 0 & & 0 & & $1(0.3)$ & \\
\hline Asian & 0 & 0 & & $1(4.8)$ & & $4(1.1)$ & \\
\hline Black or African heritage & $1(1.5)$ & 0 & & 0 & & 0 & \\
\hline Collection of data not permitted & 0 & 0 & & 0 & & $3(0.8)$ & \\
\hline Other & 7 (10.3) & 0 & & $1(4.8)$ & & $34(9.1)$ & \\
\hline White & 59 (86.8) & $25(100)$ & & 19 (90.5) & & 332 (88.8) & \\
\hline FEV $1 \%$ predicted & $74.1 \pm 14.38$ & $77.0 \pm 11.95$ & 0.23 & $75.9 \pm 15.31$ & 0.67 & $76.6 \pm 15.0$ & 0.12 \\
\hline FVC $\%$ predicted & $66.9(12.09)$ & $69.9(11.42)$ & 0.18 & $68.2(12.07)$ & 0.66 & $69.5(13.78)$ & 0.12 \\
\hline DLco \% predicted ${ }^{\text {T }}$ & $39.1 \pm 14.78$ & 40.5 (11.97) & 0.41 & $43.5 \pm 9.65$ & 0.064 & $44.1 \pm 13.69$ & 0.002 \\
\hline 6MWD m & $356 \pm 131.3$ & $372 \pm 108.3$ & 0.78 & $400 \pm 96.4$ & 0.16 & $427 \pm 115.8$ & $<0.001$ \\
\hline Residual volume \% predicted & $62 \pm 27.0$ & $55 \pm 19.4$ & 0.38 & $54 \pm 20.5$ & 0.25 & $61 \pm 22.9$ & 0.88 \\
\hline Resting $\% \mathrm{O}_{2}$ saturation & $88 \pm 20$ & $90 \pm 20.8$ & 0.039 & $92 \pm 16.5$ & 0.013 & $89 \pm 21.8$ & $<0.001$ \\
\hline $\begin{array}{l}\text { Lowest } \% \mathrm{O}_{2} \text { saturation } \\
\text { on exercise }\end{array}$ & $84 \pm 5.8$ & $88 \pm 6.3$ & 0.008 & $87 \pm 5.7$ & 0.027 & $89 \pm 5.3$ & $<0.001$ \\
\hline Cardiac output L.min ${ }^{-1}$ & $5.26 \pm 1.31$ & $5.58 \pm 1.58$ & 0.65 & $5.2 \pm 1.27$ & 0.95 & $5.42 \pm 1.49$ & 0.47 \\
\hline Cardiac index $L \cdot \mathrm{min}^{-1} \cdot \mathrm{m}^{-2}$ & $2.7 \pm 0.68$ & $2.6 \pm 0.71$ & 0.5 & $2.6 \pm 0.52$ & 0.44 & $2.8 \pm 0.77$ & 0.53 \\
\hline Mean PAP mmHg & $29.6 \pm 7.53$ & $33.4 \pm 7.35$ & 0.002 & $21.9 \pm 1.90$ & $<0.001$ & $17.7 \pm 3.82$ & $<0.001$ \\
\hline $\begin{array}{l}\text { Pulmonary vascular } \\
\text { resistance } \mathrm{mmHg} \cdot \mathrm{L}^{-1} \cdot \min \end{array}$ & $3.9 \pm 3.09$ & $2.6 \pm 1.46$ & $>0.001$ & $1.0 \pm 0.51$ & $<0.001$ & $1.8 \pm 0.90$ & $<0.001$ \\
\hline
\end{tabular}

Data are presented as mean \pm SD or $n(\%)$, unless otherwise stated. p-values were calculated by Wilcoxon rank sum test for continuous variables and Fisher's exact test for categorical variables. PAWP: pulmonary artery wedge pressure; BMI: body mass index; FEV1: forced expiratory volume in $1 \mathrm{~s}$; FVC: forced vital capacity; DLCO: diffusing capacity of the lung for carbon monoxide; 6MWD: 6-min walk distance; PAP: pulmonary arterial pressure. " : World Health Organization group; ": adjusted for haemoglobin.

and without elevation of PAWP (mean \pm SD DLCO $39.1 \pm 14.8 \%$ versus $44.1 \pm 13.7 \%(\mathrm{p}=0.002)$ and mean \pm SD 6MWD $356 \pm 131.3 \mathrm{~m}$ versus $427 \pm 115.8 \mathrm{~m}(\mathrm{p}<0.001)$, respectively).

The subjects with WHO Group $3 \mathrm{PH}$ associated with IPF had lower resting percent oxygen saturation compared to the other three categories of patients. Similarly, the subjects with group $3 \mathrm{PH}$ associated with IPF had lower minimal oxygen saturation during the course of the 6MWT compared with the other three categories of subjects (table 1).

Table 1 also shows data on baseline mean PAP, pulmonary vascular resistance (PVR) and cardiac output and cardiac index at baseline. In comparing WHO Group 3 with the other groups, PVR was higher in WHO Group 3 than in WHO Group 2; the mean \pm SD PVR for WHO Group 3 PH was 3.9 $\pm 3.09 \mathrm{mmHg} \cdot \mathrm{L}^{-1} \cdot \mathrm{min}$ versus WHO Group $2 \mathrm{PH} 2.6 \pm 1.46 \mathrm{mmHg} \cdot \mathrm{L}^{-1} \cdot \mathrm{min}(\mathrm{p}<0.001)$; cardiac output and cardiac index were not significantly different.

A histogram distribution of PAP in $5 \mathrm{mmHg}$ intervals is shown in figure 2. In addition to the $19 \%$ of the population who had $\mathrm{PH}$ (combining the subjects with WHO Group $3 \mathrm{PH}$ with the subjects who had WHO Group $2 \mathrm{PH}$ ), an additional $30 \%$ had pulmonary artery pressure values $\geqslant 20$ to $<25 \mathrm{mmHg}$. Of the $19 \%$ of subjects with $\mathrm{PH}, 15 \%$ had mean PAP $\geqslant 25$ to $<35 \mathrm{mmHg}$ and $4 \%$ had mean PAP $\geqslant 35 \mathrm{mmHg}$.

Table 2 represents Pearson correlations between baseline mean PAP, \% predicted FVC, \% predicted DLCO and $6 \mathrm{MWD}$. Most of the paired observations in the table are statistically significant. However, using Cohen's thresholds to assess the strength of the correlation, demonstrated that most of the statistically significant effects were small ( $<0.1$ negligible effect, $\geqslant 0.1$ for small effect, $\geqslant 0.3$ for moderate, $\geqslant 0.5$ for large effect). The strength of the correlation for DLCO \% predicted (adjusted for haemoglobin) was moderate in relation to the $6 \mathrm{MWD}$ and \% FVC. The effect size for mean PAP was small in relation to \% DLCO and 


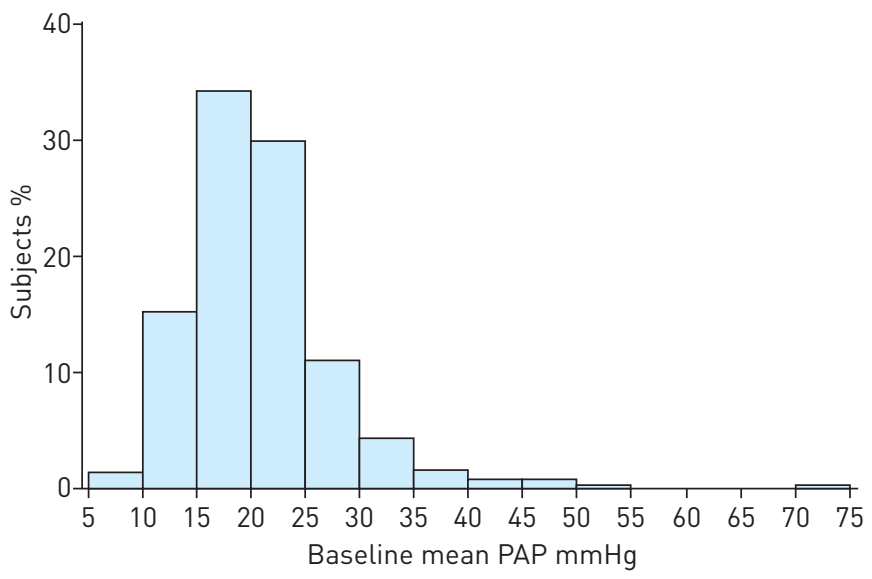

FIGURE 2 Distribution of mean pulmonary arterial pressures (mean PAP) in $\mathrm{mmHg}$. Intervals are defined as $\geqslant$ lower value to <higher value (e.g. $\geqslant 5$ to $<10 \mathrm{mmHg}$ ).

6MWD and negligible in relation to the \%FVC. The relationship between FVC/DLCO and mean PAP was statistically significant but similarly the size of the effect was small.

117 subjects (24\% of total subjects) underwent repeat RHC after 48 weeks on double-blind therapy: 44 on placebo and 74 on ambrisentan. Data are shown in table 3 for change in mean PAP, cardiac output and PVR comparing ambrisentan and placebo. Data are presented for all subjects and for the subgroup with WHO Group 3 at baseline. For all evaluable subjects, the mean \pm sD change in mean PAP over 48 weeks was a reduction of $1.1 \pm 5.99 \mathrm{mmHg}$ for ambrisentan-treated subjects and an increase of $0.4 \pm 5.85 \mathrm{mmHg}$ for subjects on placebo ( $\mathrm{p}=0.134$, Mann Whitney test). No statistically significant treatment differences were detected in any of the haemodynamic parameters. Serial haemodynamic data was available for only one subject with severe $\mathrm{PH}(\geqslant 35 \mathrm{mmHg})$ at baseline. That subject received placebo and the subject's mean PAP decreased by $2 \mathrm{mmHg}$ over 48 weeks.

Three (8\%) of 39 subjects on placebo developed PH during the 48 week treatment interval while two (3\%) of 66 subjects on ambrisentan developed PH. These differences were not statistically significant $(\mathrm{p}=0.34$, Fisher's exact test). For individual subjects, the changes in mean PAP from baseline to week 48 were as follows: for the three placebo-treated subjects mean PAP increased from 18 to $27 \mathrm{mmHg}, 17$ to $26 \mathrm{mmHg}$, and 22 to $28 \mathrm{mmHg}$, respectively; for the two ambrisentan treated subjects the increase was from 20 to $25 \mathrm{mmHg}$ and 17 to $40 \mathrm{mmHg}$, respectively. The baseline demographics of the five subjects who developed $\mathrm{PH}$ during the follow up period were similar to the baseline demographics of subjects with no $\mathrm{PH}$ and without elevation of PAWP, except for a trend towards more severe oxygen desaturation on exercise. The lowest median oxygen saturation during 6MWD was $81 \%$ in the five subjects who developed $\mathrm{PH}$ versus $89 \%$ in the 374 subjects with normal baseline haemodynamics.

In the current re-analysis of subjects with mean PAP $\geqslant 25 \mathrm{mmHg}, 90$ (28\%) of the ambrisentan-treated subjects experienced a PFS event versus 28 (17\%) of the placebo-treated subjects (HR 1.74 (95\% CI 0.68-3.57);

\begin{tabular}{|c|c|c|c|}
\hline & FVC $\%$ predicted & DLco" $\%$ predicted & 6MWD \\
\hline \multicolumn{4}{|l|}{ Mean PAP } \\
\hline Pearson coefficient & 0.047 & -0.16 & -0.20 \\
\hline p-value & 0.30 & 0.001 & $<0.001$ \\
\hline Paired observations $\mathrm{n}$ & 87 & 487 & 483 \\
\hline \multicolumn{4}{|l|}{ FVC $\%$ predicted } \\
\hline Pearson coefficient & & 0.43 & 0.20 \\
\hline$p$-value & & $<0.0001$ & $<0.0001$ \\
\hline Paired observations $\mathrm{n}$ & & 488 & 484 \\
\hline \multicolumn{4}{|l|}{ 6MWD } \\
\hline Pearson coefficient & & 0.36 & \\
\hline$p$-value & & $<0.0001$ & \\
\hline Paired observations $n$ & & 484 & \\
\hline
\end{tabular}

FVC: forced vital capacity; DLCo: diffusion capacity of the lung for carbon monoxide; 6MWD: 6-min walk distance; PAP: pulmonary arterial pressure. ${ }^{\#}$ : adjusted for haemoglobin. 
TABLE 3 Changes in haemodynamic parameters on ambrisentan and placebo between baseline and week 48

\begin{tabular}{|c|c|c|c|c|c|c|c|c|}
\hline & \multicolumn{4}{|c|}{ All subjects } & \multicolumn{4}{|c|}{ Group $3^{\#}$ PH subjects } \\
\hline & \multicolumn{2}{|c|}{ Ambrisentan } & \multicolumn{2}{|l|}{ Placebo } & \multicolumn{2}{|c|}{ Ambrisentan } & \multicolumn{2}{|l|}{ Placebo } \\
\hline & Mean士sd & $\mathbf{n}$ & Mean \pm sD & $\mathbf{n}$ & Mean \pm sD & $\mathbf{n}$ & Mean士sd & $\mathbf{n}$ \\
\hline Chat & $-1.1 \pm 5.99$ & 73 & $0.4 \pm 5.85$ & 44 & $-5.3 \pm 4.27$ & 12 & $-1.1 \pm 9.39$ & 7 \\
\hline Change in cardiac output $\mathrm{L} \cdot \mathrm{min}^{-1}$ & $0.56 \pm 1.45$ & 72 & $-0.38 \pm 1.30$ & 43 & $0.03 \pm 1.38$ & 12 & $0.44 \pm 0.9$ & 7 \\
\hline Change in PVR $\mathrm{mmHg} \cdot \mathrm{L}^{-1} \cdot \min$ & $-0.41 \pm 1.19$ & 72 & $0.14 \pm 0.79$ & 43 & $-0.70 \pm 1.31$ & 12 & $-0.51 \pm 1.56$ & 7 \\
\hline
\end{tabular}

PH: pulmonary hypertension; PAP: pulmonary arterial pressure; PVR: pulmonary vascular resistance.

\#: World Health Organization group.

$\mathrm{p}=0.28$ ). In an additional analysis that was restricted to subjects with mean PAP $\geqslant 35 \mathrm{mmHg}$, five (50\%) of the ambrisentan-treated subjects experienced a PFS event versus two (25\%) of the placebo-treated subjects (HR 1.18 (95\% CI 0.21-6.49); $\mathrm{p}=0.85$ ).

\section{Discussion}

Previous retrospective studies have demonstrated that IPF subjects with advanced disease, who undergo RHC, have a high prevalence of $\mathrm{PH}[4,5,7]$ which may progress rapidly [8]. In contrast, in the present study of IPF subjects with less severe restriction, we have characterised a subgroup constituting approximately $14 \%$ of the study population, who have WHO Group $3 \mathrm{PH}$ [9]. The presence of WHO Group $3 \mathrm{PH}$ is associated with worse gas exchange as determined by a lower DLCO and more severe oxygen desaturation on exercise. Prospective, centralised radiology review was utilised to exclude subjects with comorbid severe emphysema, while patients with physiological airway obstruction and air-trapping were excluded. Therefore, the observed PH is likely to be a consequence of IPF alone [15]. While it has been reported that there is an association between the FVC/DLCO ratio and mean PAP in patients with scleroderma, the data from this study suggests that this relationship is not clinically useful in IPF $[16,17]$.

A further $5 \%$ of the study population with IPF had WHO Group $2 \mathrm{PH}$ as evidenced by an increased PAWP and a further $4 \%$ who do not meet criteria for $\mathrm{PH}$, also had elevated PAWPs. Even though the small numbers of subjects in three of these four cohorts limit the power of statistical comparison, it appears that the subjects with WHO Group $3 \mathrm{PH}$ have the greatest degree of gas exchange abnormality. Thus, while $14 \%$ of the population had $\mathrm{PH}$ directly associated with IPF, another $9 \%$ had elevated left-ventricular pressures ( $5 \%$ with $\mathrm{PH}$ plus $4 \%$ without $\mathrm{PH}$ ), indicating subclinical left-sided cardiac disease. The level of detail on risk factors for cardiac disease was not adequate to differentiate between the risk factor profiles of Group $3 \mathrm{PH}$ and Group $2 \mathrm{PH}$ subjects, except that the latter had a slightly higher body mass index (table 1). Since patients with a left-ventricular ejection fraction of $40 \%$ were excluded from the study, this likely represents a subpopulation with heart failure and preserved ejection fraction (HFpEF or diastolic dysfunction). The finding of a significant incidence of subclinical left-ventricular disease has clinical implications in the context of a highly selected IPF population. One might speculate that the prevalence of subclinical HFpEF is even higher in all-comers with IPF. This has important implications for the differential of acute worsening of oxygenation associated with the appearance of new ground glass infiltrates in IPF patients. In addition to the $15 \%$ of the study population who met criteria for $\mathrm{PH}$, an additional $30 \%$ had mean PAP levels in $>20$ and $\leqslant 25 \mathrm{mmHg}$, a high level that is not usually seen in healthy individuals [10]. The significance of "borderline hypertension" with values of mean PAP between 21 and $24 \mathrm{mmHg}$ has been discussed in the literature but there is currently no consensus on the clinical and prognostic implications of values in this range [10]. In looking at the prevalence of left-ventricular dysfunction in IPF, it is stressed that subjects with clinically overt congestive heart failure (NYHA III and IV symptoms or LVEF <40\%) were excluded from this study. An ILD population outside of a clinical trial might be expected to have a higher prevalence of LV dysfunction [18]. Further, the exclusion of subjects with NYHA Class III and IV symptoms may also serve to decrease the number of subjects with severe, symptomatic $\mathrm{PH}$ in the current study. Although echocardiography was not part of this study, this modality is relied upon as a useful, but not sufficiently accurate, screening tool for the presence of underlying $\mathrm{PH}$. Our data support the accepted principle of not relying on echocardiography alone for the diagnosis of PH in IPF, especially when one-third of the IPF patients with PH may have a form of $\mathrm{PH}$ potentially amenable to left heart failure therapy.

An inherent strength of our study design rests in measuring haemodynamics by comparable RHC procedures 48 weeks after enrolment and thus we are able to provide accurate follow-up RHC data in 117 
subjects (44 on placebo, 73 on ambrisentan) who underwent a repeat RHC. Because of the premature termination, this represented only $24 \%$ of the randomised subjects, but still represents the largest study to date of serial RHC data in IPF patients. Only five (4\%) subjects developed PH during the study. This is in contrast to the patients with more advanced disease reported by NATHAN et al. [8] who reviewed 44 subjects with IPF in whom the presence of $\mathrm{PH}$ increased from $38 \%$ to $85 \%$ on serial RHC with an average time interval of 258 days between RHCs. This translated to an average rate of increase in mean PAP between RHCs of $3.8 \mathrm{mmHg}$ per month whereas, in the present study, the rate of increase was on average $0.17 \mathrm{mmHg}$ per month. This suggests that $\mathrm{PH}$ progresses slowly in mild, clinically stable patients but more rapidly towards the later stages of disease in those with evidence of clinical progression. There was no significant difference in treatment response as measured by serial mean PAP between those treated with ambrisentan and placebo, but the small sample size and wide confidence intervals makes it difficult to draw firm conclusions on treatment response. In future studies of this patient phenotype, the relatively low rate of progression may make it difficult to prove benefit of any intervention.

We acknowledge the limitations of this study: the ARTEMIS-IPF study was prematurely terminated for lack of efficacy with median exposure to study medication of 34 weeks; too short a duration to confidently evaluate the impact of $\mathrm{PH}$ on disease progression. At the time of study termination for lack of efficacy, an unexplained increased risk of disease progression was observed in the ambrisentan-treated subjects. In the original analysis of all subjects, ambrisentan-treated patients were more likely to meet the prespecified criteria for disease progression (90 (27.4\%) versus 28 (17.2\%) patients; $\mathrm{p}=0.010$; hazard ratio 1.74 (95\% CI 1.14-2.66)) [9]. Results of original analyses of disease progression that were stratified on the basis of baseline $\mathrm{PH}$ status were similar to those of the overall analysis (ambrisentan versus placebo hazard ratio for presence of PH, 2.42 (CI, 0.79-7.38); $\mathrm{p}=0.121$; ambrisentan versus placebo hazard ratio for absence of $\mathrm{PH}, 1.64$ (CI, 1.04-2.60); $\mathrm{p}=0.03$ ) Because the size of the $\mathrm{PH}$ subgroup limited statistical power, the authors advised against using ambrisentan to treat IPF subjects with $\mathrm{PH}$, based on the lack of efficacy and increased rate of disease progression in the overall population [9]. An additional exploratory analysis in the current study, in which the WHO Group 3 $\mathrm{PH}$ and Group $2 \mathrm{PH}$ subjects were pooled, did not show evidence of treatment benefit with ambrisentan either in subjects with mean PAP $\geqslant 25 \mathrm{mmHg}$, or in the subjects with mean PAP $\geqslant 35 \mathrm{mmHg}$, thus supporting the recommendation in the original publication to avoid using this agent in patients with IPF.

In conclusion, $14 \%$ of well-defined IPF patients with mild-to-moderate restrictive physiology had WHO Group $3 \mathrm{PH}$, while an additional 5\% had WHO Group $2 \mathrm{PH}$. A further $30 \%$ of subjects who did not meet criteria for $\mathrm{PH}$, had mean PAPs $\geqslant 20$ and $<25 \mathrm{mmHg}$ which may be suggestive of abnormal pulmonary vasculature. Subjects with IPF-PH had significantly decreased 6MWD, worse gas exchange and decreased DLCO values compared to patients with IPF without PH. In the subset of subjects who underwent repeat catheterisation, progression to $\mathrm{PH}$ over 48 weeks was uncommon. $9 \%$ of the subjects had evidence of subclinical left-ventricular dysfunction, indicating that this is an important comorbidity in patients with IPF. The observations in this limited number of patients with WHO group $3 \mathrm{PH}$ warrant further studies to understand the pathophysiogical aspects and clinical outcomes of the pulmonary vasculopathy associated with IPF.

\section{References}

1 Raghu G, Collard HR, Egan JJ, et al. An official ATS/ERS/JRS/ALAT statement: idiopathic pulmonary fibrosis: evidence-based guidelines for diagnosis and management. Am J Respir Crit Care Med 2011; 183: 788-824.

2 Shlobin OA, Nathan SD. Pulmonary hypertension secondary to interstitial lung disease. Expert Rev Respir Med 2011; 5: 179-189.

3 Seeger W, Adir Y, Barberà JA, et al. Pulmonary hypertension in chronic lung disease. J Am Coll Cardiol 2013; 62: D109-D116.

4 Rivera-Lebron BN, Forfia PR, Kreider M, et al. Echocardiographic and hemodynamic predictors of mortality in idiopathic pulmonary fibrosis. Chest 2013; 144: 564-570.

5 Lederer DJ, Borczuk AC, Kawut SM. Pulmonary hypertension in idiopathic pulmonary fibrosis. Chest 2007; 132: 998-1006.

6 Corte TJ, Wort SJ, Wells AU. Pulmonary hypertension in idiopathic pulmonary fibrosis: a review. Sarcoidosis Vasc Diffuse Lung Dis 2009; 26: 7-19.

7 Shorr AF, Wainright JL, Cors CS, et al. Pulmonary hypertension in patients with pulmonary fibrosis awaiting lung transplant. Eur Respir J 2007; 30: 715-721.

8 Nathan SD, Shlobin OA, Ahmad S, et al. Serial development of pulmonary hypertension in patients with idiopathic pulmonary fibrosis. Respiration 2008; 76: 288-294.

9 Raghu G, Behr J, Brown KK, et al. Treatment of idiopathic pulmonary fibrosis with ambrisentan: a parallel, randomized trial. Ann Intern Med 2013; 158: 641-649.

10 Hoeper MM, Bogaard HJ, Condliffe R, et al. Definitions and diagnosis of pulmonary hypertension. J Am Coll Cardiol 2013; 62: Suppl. 25, D42-D50.

11 American Thoracic Society. Idiopathic pulmonary fibrosis: diagnosis and treatment. International consensus statement. American Thoracic Society (ATS), and the European Respiratory Society (ERS). Am J Respir Crit Care Med 2000; 161: 646-664.

12 Galie N, Hoeper MM, Humbert M, et al. Guidelines for the diagnosis and treatment of pulmonary hypertension. The Task Force for the Diagnosis and Treatment of Pulmonary Hypertension of the European Society of 
Cardiology (ESC) and the European Respiratory Society (ERS), endorsed by the International Society of Heart and Lung Transplantation (ISHLT). Eur Heart J 2009; 30: 2493-2537.

13 Seeger W, Adir Y, Barberà JA, et al. Pulmonary hypertension in chronic lung diseases. J Am Coll Cardiol 2013; 62: Suppl. 25, D109-D116.

14 Cohen J. Statistical Power Analysis for the Behavioral Sciences 2nd Edn. Hillsdale, Lawrence Erlbaum, 1988.

15 Mejía M, Carrillo G, Rojas-Serranfo J, et al. Idiopathic pulmonary fibrosis and emphysema: decreased survival associated with severe pulmonary arterial hypertension. Chest 2009; 136: 10-15.

16 Steen V, Thomas A, Medsger J. Predictors of isolated pulmonary hypertension in patients with systemic sclerosis and limited cutaneous involvement. Arthritis Rheum 2003; 48: 516-522.

17 Chang B, Schachna L, White B, et al. Natural history of mild-moderate pulmonary hypertension and the risk factors for severe pulmonary hypertension in scleroderma. J Rheumatol 2006; 33: 269-274.

18 Hyldgaard C, Hilberg O, Bendstrup E. How does comorbidity influence survival in idiopathic pulmonary fibrosis? Respir Med 2014; 108: 647-653. 